\title{
Multiple risk factors contribute to childhood stunting in Karnataka, India
}

\author{
Srilakshmi M. Raj ${ }^{1,2, *}$, Ruwanthi Ekanayake ${ }^{3}$, Meenakshi Bhat ${ }^{2}$, \\ Jayarama Kadandale ${ }^{2}$ and Prabhu L. Pingali ${ }^{1}$ \\ ${ }^{1}$ Tata Cornell Institute, Cornell University, Ithaca, NY 14853, USA \\ ${ }^{2}$ Centre for Human Genetics, Electronics City Phase 1, Electronic City, Bengaluru 560 100, India \\ ${ }^{3}$ Department of Biology and Society, Cornell University, Ithaca, NY 14853, USA
}

\begin{abstract}
Childhood stunting remains a prominent metric in the health and development of modern India. In Karnataka, India, districts vary substantially in stunting prevalence. Here we take a close look at the nature of childhood stunting in the state: its epidemiology, genetics, biology, nutritional basis, environmental contribution, policy and field-based understanding. We explore how these factors interact with one another, and suggest ways to target modifiable risk factors for stunting (e.g. nutrition, exposure to infectious diseases, sanitation), which may influence the impact of non-modifiable risk factors (e.g. congenital factors, climate). We suggest that programmes designed to reduce stunting prevalence should accommodate these complex interactions, which could translate to more substantial impact on child health outcomes.
\end{abstract}

Keywords: Childhood stunting, environment, genetics, public health, risk factors.

\section{Childhood stunting in Karnataka}

CHILDHood stunting is defined as reduced linear growth that is two standard deviations below the global standard, for children up to 5 years of age $\mathrm{e}^{1,2}$. Stunted children show poor academic performance, which eventually results in increased rates of not completing secondary school education $^{3}$, and these effects are more pronounced the earlier in life stunting occurs ${ }^{4}$. If stunting is reversed later in childhood owing to changes in diet or environment, in a phenomenon called 'catch-up growth', formerly stunted children are at higher risk for non-communicable diseases such as obesity, diabetes, hypertension and cardiovascular disease $\mathrm{e}^{3,5}$. The long-term consequences of stunting are irreversible by 2 years of age ${ }^{6}$.

The state of Karnataka is located in South India, and with 61 million people in 30 districts, it constitutes $5 \%$ of the country's population ${ }^{7}$. Based on the socio-demographic index (SDI), Karnataka is classified as a "middle SDI' state ${ }^{8}$. It also belongs to the 'Higher Middle Epidemiological Transition Level' group of states in India'.

\footnotetext{
*For correspondence. (e-mail: sri@chg.res.in)
}

Overall, Karnataka fares better than many other Indian states on childhood stunting metrics, experiencing a reduction in stunting prevalence from $43.7 \%$ to $36.2 \%$ between 2006 and 2016 (ref. 10). While this is a step in the right direction, it has the greatest coefficient of variation for childhood stunting among the middle SDI states ${ }^{11}$. The high coefficient of variation for childhood stunting in India reflects high variability in stunting prevalence across Karnataka; 9 out of the 30 districts have stunting prevalence $>40 \%$, with Koppal and Yadgir districts showing $>55.5 \%$ stunting prevalence ${ }^{12}$. In contrast, Mandya district has a stunting prevalence of only $18 \%$, half the state-wide average and three times lower than that of the hardest hit states. This variation indicates the potential for different interactions between stunting risk factors across different districts in Karnataka.

\section{Risk factors for childhood stunting}

Of the myriad factors that contribute to child growth outcomes, we identify a set of non-modifiable and modifiable factors that contribute to stunting outcomes in Karnataka. The non-modifiable factors we discuss are climate and congenital (genetic) factors, and the modifiable factors are nutrition and infectious diseases/diarrhoea. Interactions between both contribute to child growth outcomes in Karnataka and elsewhere.

\section{Climate}

One reason for the diversity in stunting prevalence across Karnataka could be regional climate and environment. With just over $190,000 \mathrm{~km}^{2}$ area, Karnataka has at least ten separate agro-climate zones where climate, soil, elevation, dominant cropping season and crops grown differ $^{13}$. For instance, annual rainfall ranges from 453 to $717.7 \mathrm{~mm}$ in the central dry zone, and 3010.9 to $4694.4 \mathrm{~mm}$ in the coastal zone, representing nearly ten times higher rainfall in some places within the state. Combined with soil type and differences in elevation, these vast differences in rainfall also contribute to major differences in crop production and thus dietary habits. 
For instance, in the central dry zone where the dominant cropping season is kharif (monsoon season) and the major soil type is red sandy loam, ragi (finger millet), jowar (sorghum), pulses and oilseeds are grown. In contrast, in the coastal zone, where monsoon is the dominant cropping season and the major soil type is red lateritic, rice, pulses and sugarcane are the primary crops ${ }^{13}$.

Climate change is widely considered to be a threat to food security and nutrition around the world. Climate itself can shape childhood growth trajectories. Out of every 1000 births in India, two children die owing to high temperature when in gestation, due in part to reduced agricultural yield, reduced wages and increased disease prevalence like diarrhoea. This relationship only exists in rural India ${ }^{14}$.

Similarly, variability in climate at a sub-country or state level (e.g. Karnataka) can also influence food security and health outcomes by directly influencing crop production and food availability ${ }^{15}$. Most recently, it has been demonstrated that areas that experience minor to severe drought, as well as periods of severe rainfall, are highly vulnerable to childhood stunting ${ }^{16,17}$. Some factors that could reduce the impact of drought on stunting outcomes include effective government, nutritional diversity in local agricultural systems, increased importing and production of staple crops, and an emphasis on irrigated agriculture $^{17}$. While this study was based on an international dataset for countries with very different agro-climate and other variables, there are implications that apply to Karnataka. The districts of Karnataka with lower rainfall also have the highest rates of childhood stunting ${ }^{18}$.

\section{Congenital/clinical risk factors}

Congenital anomalies, by definition appearing prior to birth, result in the death of approximately 303,000 children worldwide ${ }^{19}$. Although nearly $50 \%$ have unknown causes, many of the known causes are genetic and show population-specific prevalence and aetiology. It is also known that approximately $94 \%$ of severe congenital anomalies occur in low- and middle-income countries, pointing to socioeconomic factors as a major contributor. Such factors may impede access to nutritious food, sanitation, in utero infectious disease prevention, clean environment and healthcare, which demonstrably influence child growth trajectories as well.

India may have the highest number of infants born with birth defects in the world ${ }^{20,21}$, with an estimated 1 in 50 children showing some form of congenital malformation $^{22}$. Indians may be particularly prone to congenital risk factors due to high levels of consanguinity, particularly in the Muslim and Hindu populations. In South India, $20-30 \%$ of Hindu marriages are consanguineous. Notably, consanguinity has been linked to higher rates of neural tube defects in Davangere, Karnataka ${ }^{23,24}$.
Beta thalassemia is an example of a congenital disorder in which many affected children show stunted growth ${ }^{25}$. In India, the average frequency of the disorder is $3-4 \%$, with the highest prevalence appearing in the Jain population in Bengaluru (9.6\%), Karnataka ${ }^{21,26}$. Other genetically linked metabolic disorders which could also influence child growth show regional differences in India. In Karnataka, the most prevalent amino acid disorders include: tyrosinaemia, maple syrup urine disease, phenylketonuria and generalized amino acidurias ${ }^{27}$. In North India, however, and among other communities, other metabolic disorders are more prevalent, such as hyperglycinaemia, homocystinuria and alkaptonuria ${ }^{21,28}$.

Cataloguing congenital anomalies among communities in Karnataka will help develop a better picture of biological challenges faced by children who are vulnerable to adverse growth outcomes, and also provide more targeted means of improvement.

\section{Nutrition}

Malnutrition is considered to be the leading cause of childhood stunting and childhood mortality in all states in India ${ }^{8}$. According to data from the National Family Health Survey-4 (NFHS-4), Karnataka also shows variability in malnutrition metrics. Some traits are uniformly distributed across the state: anaemia among women of reproductive age and childhood wasting prevalence are uniformly high across Karnataka. However, severe childhood wasting prevalence and stunting prevalence show a pattern with high prevalence in north Karnataka and lower prevalence in the coastal districts. Similarly, Karnataka shows lower iodized salt consumption than other states $(86.8 \%$ compared to $>90 \%$ in most other states), but high variability within the state: $66.4 \%$ of households in Chikkaballapura district report iodized salt usage compared to $96.3 \%$ of households in Belgaum district $^{29}$

Environmental differences, as suggested in the previous section, may contribute to these trends. Karnataka's coastal region, like other coastal regions, has fairly high fish consumption, which should translate to low-fat, highprotein and high-mineral diets. However, analyses in 2015 suggest that while the proportion of household food budgets spent on fish has increased, total fish consumption has decreased, as households cannot keep up with rising fish prices ${ }^{30}$. This may provide an explanation for variation in indicators of malnutrition in the state. Furthermore, a 2015 nutritional analysis in Koppal district revealed that urban households consume fewer cereals and more pulses than rural households ${ }^{31}$, indicating that protein deficiencies might be more common in rural areas. Agroeconomics may play a role in this variation, as increased rural cereal consumption has been linked to higher prices of dairy, meat and vegetables. Consumption of coarse grains, which are important sources of calcium, 


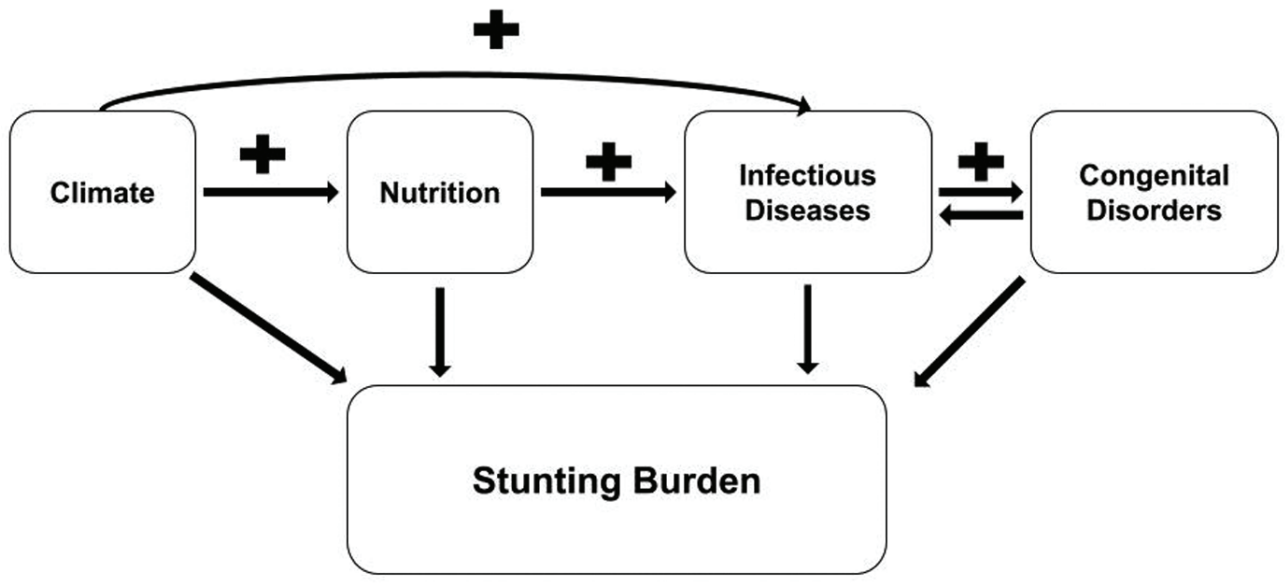

Figure 1. Potential interaction of stunting risk factors in Karnataka, India.

iron and phosphorus (particularly for vegetarian households $)^{32}$, has decreased overall in both rural and urban $\operatorname{areas}^{31}$.

The Government of Karnataka instituted various interventions and prenatal and postnatal care measures between 2006 and 2016 to improve nutrition outcomes ${ }^{10}$. These interventions were broad-ranging, including antenatal care and food, iron and folic acid supplementation during pregnancy, administration of oral rehydration salts for diarrhoea treatment, and updated midday-meal schemes that prioritized vitamin A and increased food supplementation. The midday meal scheme, introduced nationwide by the Government of India in 1995, was shown to reduce stunting in children in rural Karnataka for the 7-12-yr age bracket ${ }^{33}$. However, these changes may not be sufficient. Until recently, the daily menu served to schoolgoing children included lentil and vegetable stew (rice and sambar) five days a week, and a lentil and rice-based meal (bisibelebath), or semolina/rice flour porridge (upma) on Saturdays ${ }^{34}$. A recent study in Karnataka on school-going children showed that adding more millets to existing midday meal schemes could provide a statistically significant reduction in stunting and improvements in body mass index ${ }^{35}$. The state appears to have heeded this advice by promising in late 2019 to increase protein amounts and dietary diversity in midday meals for children in classes 1 to 5 (ref. 36).

\section{Infectious diseases/diarrhoea}

Diarrhoea is the second leading cause of death in children under 5 years of age, killing over half a million children in this age group each year and contributing substantially to malnutrition ${ }^{37}$. In India in particular, diarrhoea is the third leading cause of childhood mortality ${ }^{38}$. In 2014, the Karnataka government introduced the pro-sanitation programme Swachh Bharat Mission, the world's largest toilet-building initiative ${ }^{39}$, as a response to diarrhoeal dis- ease. Studies have found that the Swachh Bharat Mission may have led to a reduction in acute diarrhoeal disease outbreaks, but that these relationships need to be further examined to determine areas for improvement ${ }^{38-40}$. For example, rotavirus infection, a common cause of diarrhoeal disease in India, remains at high prevalence in the country $^{41}$. In southern Karnataka, despite lower diarrhoeal disease, rotavirus diarrhoea among hospitalized children was found to be high ${ }^{42}$. Diarrhoeal disease could be further curbed by introducing an affordable rotavirus vaccine into the national immunization scheme ${ }^{38}$. Other perinatal infections that have been reported in India include cytomegalovirus, rubella, toxoplasmosis, herpes simplex, chicken pox, parvovirus, hepatitis B and E, and $\mathrm{HIV}^{21}$.

\section{Risk factor interactions and variation}

Climate, nutrition, infectious diseases and congenital disorders are some of the most significant contributors to stunting burden in Karnataka, but they rarely work in isolation. Stunting risk has long been known to be multifactorial, and risk factors show both additive and interaction effects. Studies examining potential 'subgroups' among stunted Indian children suggest that they can be categorized into discrete clusters of risk 'types', where factors like income, age and sanitation practices act together in different ways to produce varied stunting outcomes ${ }^{43}$. Analysis of complementary feeding practices also reveals that cultural differences in feeding practices can interact with epigenetic mechanisms, maternal health and infections, among other factors, to influence stunting risk differently depending on household context ${ }^{44}$.

The modifiable risk factors to stunting in Karnataka have the potential to influence stunting burden through mediating the effects of non-modifiable risk factors, and vice versa (Figure 1). For example, low rainfall in some areas may translate to less diverse cropping, lowering the 


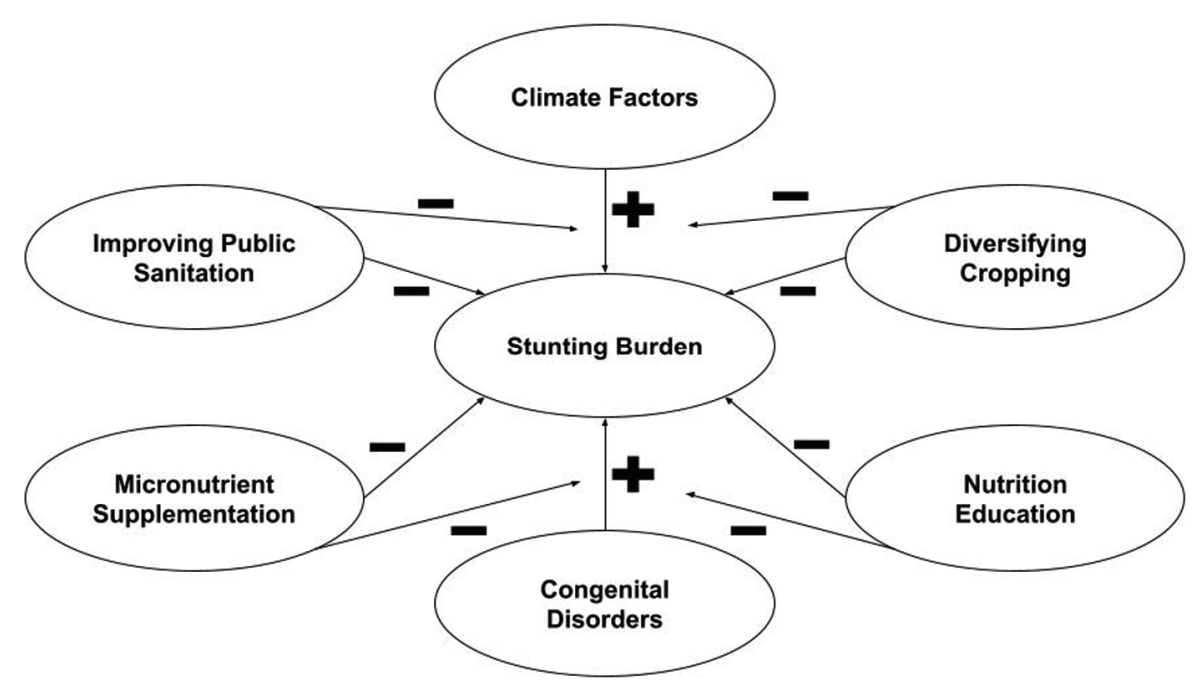

Figure 2. Targeted interventions of modifiable risk factors in Karnataka which have the potential to decrease the impact of nonmodifiable risk factors.

abundance of micronutrient-rich foods. This nutritional deficiency may worsen the impact of pre-existing metabolic conditions and further reinforce childhood stunting risk. This in turn may expose children to greater risk of infectious diseases, even in dry environments, as their immune systems are compromised by metabolic and nutritional deficiencies.

\section{Contextualize stunting interventions}

These complex interactions may result in a tendency to take an 'everything but the kitchen sink approach' to alleviate stunting, because the interrelationships may be too difficult to disentangle. However, these interactions must be specifically evaluated because they can inform more targeted interventions that are both cost- and resource-efficient as well as effective at a regional level. In the case of Karnataka, recognizing the impacts of nonmodifiable risk factors (e.g. climate and congenital disorders) on modifiable ones (e.g. nutrition and exposure to infectious diseases), and vice versa, allows extra resources to be devoted to interventions and preventative measures like nutrition education, more diverse agricultural practices, micronutrients supplementation, and stronger home and public sanitation efforts (Figure 2). These have the potential to not only decrease their own contribution to stunting burden, but also that of the nonmodifiable factors, particularly as issues like climate change threaten local environmental resources.

\section{Conclusion}

It is clear that stunting, and other aspects of child growth, are affected by many different factors, and that a multipronged approach is required to solve the problem. There is a growing body of evidence that population-level variation, including environmental, climatological, biological and/or genetic variation must be taken into account when forming policies related to child health outcomes. These variations may interact with one another in complex ways, ultimately contributing to the childhood stunting burden. These interactions must be disentangled in order to design targeted and effective health interventions. The Karnataka government is taking some of the proposed policy initiatives into consideration and has implemented many schemes which have resulted in a reduction in stunting prevalence, but more needs to be done ${ }^{10}$. Biologists, geneticists and clinicians must consider variation in stunting risk factors across the state, and liaise with policymakers to determine interacting risk factors in different contexts. These efforts can help design more precise stunting interventions. Success in these efforts can have wide-ranging implications across India, setting the foundation for more localized and contextual stunting programmes.

1. de Onis, M. and Branca, F., Childhood stunting: a global perspective. Matern. Child Nutr., 2016, 12(Suppl. 1), 12-26.

2. World Health Organization. WHO Child Growth Standards: Training Course on Child Growth Assessment. WHO Press, Geneva, Switzerland. 2008.

3. Adair, L. S., Discussion on economic drivers and consequences of stunting. Nestle Nutr. Inst. Workshop Ser., 2013, pp. 143-146.

4. Mendez, M. A. and Adair, L. S., Severity and timing of stunting in the first two years of life affect performance on cognitive tests in late childhood. J. Nutr., 1999, 129, 1555-1562.

5. Guerrant, R. L., DeBoer, M. D., Moore, S. R., Scharf, R. J. and Lima, A. A. M., The impoverished gut - a triple burden of diarrhoea, stunting and chronic disease. Nat. Rev. Gastroenterol. Hepatol., 2013, 10, 220-229.

6. UNICEF, Stop Stunting UNICEF India; https://www.unicef.org/ india/what-we-do/stop-stunting (accessed on 30 June 2021).

7. Census of India 2011: Karnataka. Directorate of Census Operations, Karnataka. 2011. 


\section{REVIEW ARTICLE}

8. Swaminathan, S. et al., India state-level disease burden initiative malnutrition collaborators, The burden of child and maternal malnutrition and trends in its indicators in the states of India: the Global Burden of Disease Study 1990-2017. Lancet Child Adolesc Health, 2019.

9. Dandona, L. et al., Nations within a nation: variations in epidemiological transition across the states of India, 1990-2016 in the Global Burden of disease study. Lancet, 2017, 390, 2437-2460.

10. Pampackal, A. J., Nguyen, P. H., Avula, R., Tran, L. M. and Menon, P., Improving Nutrition in Karnataka: Insights from Examining Trends in Outcomes, Determinants and Interventions between 2006 and 2016, International Food Policy Research Institute, 2017.

11. India state-level disease burden initiative CGF collaborators, mapping of variations in child stunting, wasting and underweight within the states of India: the Global Burden of Disease Study 2000-2017. EClinical Med., 2020, 22, 100317.

12. Mohanty, S. K., Mishra, N. R., Khan, J., Vasishtha, G. and Mishra, U. S., State of health in the districts of India. In The Demographic and Development Divide in India: A District-Level Analyses (eds Mohanty, S. K., Mishra, U. S. and Chauhan, R. K.), Springer Singapore, Singapore, 2019, pp. 329-373.

13. Ramakrishna, T. V. and Kamakshi, G., Bioresource Potential of Karnataka Technical Report 109, Centre for Ecological Sciences, Indian Institute of Science, Bangalore, 2005.

14. Banerjee, R. and Maharaj, R., Heat, infant mortality, and adaptation: evidence from India. J. Dev. Econ., 2020, 143, 102378.

15. Schlenker, W. and Lobell, D. B., Robust negative impacts of climate change on African agriculture. Environ. Res. Lett., 2010, 5, 014010 .

16. Shively, G., Sununtnasuk, C. and Brown, M., Environmental variability and child growth in Nepal. Health Place, 2015, 35, 37-51.

17. Cooper, M. W. et al., Mapping the effects of drought on child stunting. Proc. Natl. Acad. Sci. USA, 2019, 116, 17219-17224.

18. Raj, S. M., Ekanayake, R., Crowley, K., Bhat, M., Kadandale, J. and Pingali, P. L., Risk factors in childhood stunting in Karnataka state vary by geography. Curr. Sci., 2021, in press.

19. World Health Organization. Congenital anomalies, 2020; https:// www.who.int/news-room/fact-sheets/detail/congenital-anomalies (accessed on 30 June 2021).

20. Christianson, A. L., Howson, C. P. and Modell, B., Global Report on Birth Defects: The Hidden Toll of Dying and Disabled Children, March of Dimes Birth Defects Foundation, White Plains, NY, 2006.

21. World Health Organization, Birth Defects in South East Asia: A Public Health Challenge, WHO, New Deli, India, 2013.

22. Verma, I. C. and Kumar, D., Epidemiology of genetic diseases in the Indian subcontinent. Genomics and Health in the Developing World, Oxford University Press, Oxford, UK, 2012.

23. Kulkarni, M. L., Mathew, M. A. and Ramachandran, B., High incidence of neural-tube defects in South India. Lancet, 1987, 1 1260 .

24. Kulkarni, M. L., Mathew, M. A. and Reddy, V., The range of neural tube defects in southern India. Arch. Dis. Child., 1989.

25. Tienboon, P., Sanguansermsri, T. and Fuchs, G. J., Malnutrition and growth abnormalities in children with beta thalassemia major. Southeast Asian J. Trop. Med. Public Health, 1996, 27, 356-361.

26. Mohanty, D. et al., Prevalence of $\beta$-thalassemia and other haemoglobinopathies in six cities in India: a multicentre study. J. Commun. Genet., 2013, 4, 33-42.

27. Appaji Rao, N., Radha Rama Devi, A., Savithri, H. S., Venkat Rao, S. and Rezvani, I., Defects in metabolism of amino acids. Behrman Bittles AH. Neonatal screening for aminoacidemias in Karnataka, South India. Clin. Genet., 1988, 34, 60-63.
28. Kaur, M., Das, G. P. and Verma, I. C., Inborn errors of amino acid metabolism in north India. J. Inherit. Metab. Dis., 1994, 17, 230 233.

29. Kumar, A., Kalra, S. and Unnikrishnan, A. G., Iodized salt in India: Insights from National Family Health Survey-4. Thyroid Res. Practice, 2016, 13, 49.

30. Ravikanth, L. and Kumar, K. S. K., Caught in the 'Net': fish consumption patterns of coastal regions in India. Madras School of Economics, 2015, 1-30.

31. Kiresur, V. R. and Chourad, R., Nutrient intake optimization in Karnataka: a linear programming approach. Agric. Econ. Res. Rev., 2015, 28, 147-156.

32. Kaur, K. D., Jha, A., Sabikhi, L. and Singh, A. K., Significance of coarse cereals in health and nutrition: a review. J. Food Sci. Technol., 2014, 51, 1429-1441.

33. Minj, C., Ramakrishna Goud, B., James, D., Furruqh, F., Mohammad, A. and Bhavan, R. K., Impact of School mid day meal program on the Nutritional status of children in a rural area of South Karnataka, India, 2014.

34. Mirajkar, B. C., Ravindra, U. and Narayanaswamy, T., An overview of mid-day meal scheme in Karnataka. Food Sci. Res. J., 2016, 7(2), 319-326.

35. Anitha, S. et al., Acceptance and impact of millet-based mid-day meal on the nutritional status of adolescent school going children in a Peri urban region of Karnataka state in India. Nutrients, 2019, 11(9), 2077.

36. Kulkarni, T., An all-new midday meal menu for students in Karnataka. The Hindu, 27 October 2019.

37. WHO, Diarrhoeal Disease, World Health Organization, Geneva, Switzerland, 2017.

38. Lakshminarayanan, S. and Jayalakshmy, R., Diarrheal diseases among children in India: current scenario and future perspectives. J. Nat. Sci. Biol. Med., 2015, 6, 24-28.

39. Dandabathula, G., Bhardwaj, P., Burra, M., Prasada Rao, P. V. and Rao, S., Impact assessment of India's Swachh Bharat Mission - Clean India Campaign on acute diarrheal disease outbreaks: yes, there is a positive change. J. Family Med. Primary Care, 2019, 1202.

40. Ramanathan, M. and Vijayan, B., Covariates of diarrhoea among under-five children in India: are they level dependent? PLOS ONE, 2019, 14, e0221200.

41. Nilima, K. A., Shetty, K., Unnikrishnan, B., Kaushik, S. and Rai, S. N., Prevalence, patterns, and predictors of diarrhea: a spatialtemporal comprehensive evaluation in India. BMC Public Health, 2018, 18, 1288.

42. Shetty, R. S., Kamath, V. G., Nayak, D. M., Hegde, A. and Saluja, T., Rotavirus associated acute gastroenteritis among under-five children admitted in two secondary care hospitals in southern Karnataka, India. Clin. Epidemiol. Global Health, 2017, 5, 28-32.

43. Green, M. A., Corsi, D. J., Mejía-Guevara, I. and Subramanian, S. V., Distinct clusters of stunted children in India: an observational study. Matern. Child Nutr., 2018.

44. Stewart, C. P., Iannotti, L., Dewey, K. G., Michaelsen, K. F. and Onyango, A. W., Contextualising complementary feeding in a broader framework for stunting prevention. Matern. Child Nutr., 2013, 9(Suppl. 2), 27-45.

ACKNOWLEDGEMENT. We thank Prof. H. Sharat Chandra, Centre for Human Genetics, Bengaluru for his thorough review and comments on the manuscript.

Received 30 October 2020; revised accepted 4 June 2021

doi: $10.18520 / \mathrm{cs} / \mathrm{v} 121 / \mathrm{i} 3 / 360-364$ 